\title{
SETS OF COINCIDENCE* POINTS \\ ON THE NON-SINGULAR CUBICS OF A SYZYGETIC SHEAF ${ }^{\dagger}$
}

BY

M. B. PORTER

I.

If $C_{3}=0$ be any non-singular cubic and

$$
u=\int_{a b .}^{x y} d u
$$

be an everywhere finite integral attached to this curve, the point $(a, b)$ being a point of inflexion, the necessary and sufficient condition that $3 \mathrm{~m}$ points $P_{1} \ldots P_{3 m}$ of $C_{3}$ lie also on an $m$-ic is given by ABEL's theorem, namely :

$$
\sum_{1}^{3 m} u_{i} \equiv 0 \quad\left(\bmod . \omega, \omega^{\prime}\right) \text {, }
$$

where $\omega$ and $\omega^{\prime}$ are the moduli of periodicity of $u$ and

$$
u_{i}=\int_{a b}^{x_{i} y_{i}} d u \quad(i=1, \cdots, 3 m),
$$

the points $P_{i}$ being the points $\left(x_{i}, y_{i}\right)$.

If $3 m-s-1$ of these points $P_{i}$ be taken as fixed and we suppose the $m$-ic to have a contact of order $s-1$ at $u_{1}$, the $m$-ic will still cut $C_{3}$ at a point $u_{2}$ dependent on $u_{1}$ and by (1) we have :

$$
s u_{1}+u_{2} \equiv c\left(\bmod . \omega, \omega^{\prime}\right),
$$

where $c$ denotes the negative of the sum of the $u$ 's of the arbitrarily chosen fixed points.

We consider the following sequence of $n$ such congruences :

$$
\left.\begin{array}{c}
s u_{1}+u_{2} \equiv c \\
s u_{2}+u_{3} \equiv c \\
\vdots \\
s u_{n}+u_{n+1} \equiv c
\end{array}\right\}\left(\bmod \omega, \omega^{\prime}\right)
$$

* Points where a cubic can have an eighth order contact with oubics of the syzygetio sheat are called by HaLPHEN coincidence points of the cubic.

† Presented to the Society at the New York meeting, June 29, 1900, under a different title. Received for publication November 16, 1900. 
if we require that $u_{n+1} \equiv u_{1}$, i. e., that the curvilinear polygon of points of parameters $u_{1} \cdots u_{n+1}$ be a closed polygon,* the necessary and sufficient condition will be :

$$
\left[s^{n}-(-1)^{n}\right] u_{1} \equiv c^{\prime}\left(\bmod . \omega, \omega^{\prime}\right) .
$$

In case $m=s=1$, we have a rectilinear polygon of $n$ sides inscribed and circumscribed in $C_{3}$, (2) becoming in this case,

$$
\left.\left[2^{n}-(-1)^{n}\right] u_{1} \equiv 0 \text { (mod. } \omega, \omega^{\prime}\right) \text {. }
$$

The number of actual $n$-gons is given by a formula of PicQuet* and it is evident from the nine perspective transformations of $C_{3}$ into itself that any one of these $n$-gons will be perspective with some other $n$-gon relative to an inflexion point.

HALPHEN $\ddagger$ has investigated the points $u=\left(n^{\prime} \omega+n^{\prime \prime} \omega^{\prime}\right) / 3 m$ which contain as special cases the vertices of these rectilinear $n$-gons for the non-singular cubics of the syzygetic sheaf $x^{3}+y^{3}+z^{3}+6 a x y z=0$, and finds that the locus of these points as $a$ varies, which is evidently a combinant of the sheaf, consists of eight irreducible curves when $m$ is divisible by 3 , and of nine such curves when $m$ is not so divisible.

\section{II.}

It is the purpose of this section to consider certain geometrical relations that subsist between an inflexion triangle and its associated group of in- and circum. scribed rectilinear triangles. $\quad B y\left(2^{\prime}\right)$ the number of such triangles is 24 .

* The Schliessungsproblem here suggested is a slight generalization of that considered by Picquet (Journal de l'École Polytechnique, vol. 54, 1884). The method of enumeration used by PICQUeT is of course applicable here, but the number-theoretic results arrived at are completely general. Thus for $n$ prime the number of $n$-gons on the part of the curve corresponding to $\omega$ will be :

$$
\frac{s^{n}-s}{n},
$$

since $s+1$ values of $u$ must be rejected as corresponding to the s-contact points. In case $n$ be not prime, to obtain the number of $n$-gons it would be necessary to reject besides the $s+1$ $s$-contact points all solutions corresponding to polygons whose number of sides divides $n$. Denoting by $\phi(s, m)$ the number of vertices of such $m$-gons on the part of the curve corresponding to $\omega$ we have :

$$
8^{n}-(-1)^{n}-8-1=\sum_{m^{\prime}} \phi\left(s, m^{\prime}\right) \quad\left(1<m^{\prime} \leqq n\right),
$$

where the summation is for the different factors $m^{\prime}$ of $n$.

By [B] for $n \neq 2$ we get :

$$
\phi(s, n)=s^{n}-\sum s^{\frac{n}{a_{i}}}+\sum s^{\frac{n}{a_{i} a_{j}}}-\cdots \pm s^{\frac{n}{a_{1} a_{2} \cdots \cdot a_{\nu}}}=F(s, n),
$$

$a_{1} a_{2} \cdots a_{\nu}$ denoting the different prime factors of $n$. For $n=2$ we have $\phi=F-2$.

The expressions [A] and [B] may be regarded as establishipg Fermat's theorem and ito generalization.

$\ddagger$ Recherches sur les courbes planes du troisième degré, Mathematische Annalen, vol. 15 . 
These twenty-four triangles can be divided into four groups of six each, each group being associated with one of the four inflexion triangles. Further the six triangles of any group can be paired in nine ways so that each pair shall be perspective in three ways, the centers of perspectivity for each pair being the three inflexion points lying on a side of the associated inflexion triangle.

Thus for the inflexion triangle whose sides may be denoted by

$$
\left(\omega+\frac{n \omega^{\prime}}{3}, \frac{\omega+n \omega^{\prime}}{3}, \frac{2 \omega+n \omega^{\prime}}{3}\right) \quad(n=0,1,2),
$$

the scheme is as follows:

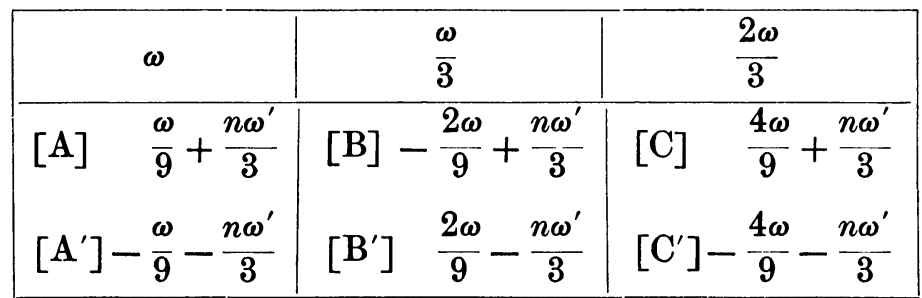

\begin{tabular}{|c|c|c|}
\hline$\omega+\frac{\omega^{\prime}}{3}$ & $\frac{\omega+\omega^{\prime}}{3}$ & $\frac{2 \omega+\omega^{\prime}}{3}$ \\
\hline$\frac{\omega}{9}+\frac{n \omega^{\prime}}{3}$ & $-\frac{2 \omega}{9}+\frac{n \omega^{\prime}}{3}$ & $\frac{4 \omega}{9}+\frac{n \omega}{3}$ \\
$-\frac{\omega}{9}-\frac{(n+1) \omega^{\prime}}{3}$ & $\frac{2 \omega}{9}-\frac{(n+1) \omega^{\prime}}{3}$ & $-\frac{4 \omega}{9}-\frac{(n+1) \omega^{\prime}}{3}$ \\
\hline
\end{tabular}

\begin{tabular}{|c|c|c|}
\hline$\omega+\frac{2 \omega^{\prime}}{3}$ & $\frac{\omega+2 \omega^{\prime}}{3}$ & $\frac{2 \omega+2 \omega^{\prime}}{3}$ \\
\hline$\frac{\omega}{9}+\frac{n}{3} \omega^{\prime}$ & $-\frac{2 \omega}{9}+\frac{n \omega^{\prime}}{3}$ & $\frac{4 \omega}{9}+\frac{n \omega^{\prime}}{3}$ \\
$\frac{\omega}{9}-\frac{(n-1) \omega^{\prime}}{3}$ & $\frac{2 \omega}{9}-\frac{(n-1) \omega^{\prime}}{3}$ & $-\frac{4 \omega}{9}-\frac{(n-1) \omega^{\prime}}{3}$ \\
\hline
\end{tabular}

where $A B C$ is perspective with $A^{\prime} B^{\prime} C^{\prime}$, with $B^{\prime} C^{\prime} A^{\prime}$, and with $C^{\prime} A^{\prime} B^{\prime}$, the centers of perspectivity being $\omega, 2 \omega / 3$, and $\omega / 3$ respectively. Thus the nine intersections of the sides of these two triangles lie three by three on the harmonic polar of $\omega, 2 \omega / 3, \omega / 3$.

Referred to one of these in-circumscribed triangles, as $\omega / 9,-2 \omega / 9,4 \omega / 9$, the equation of $C_{3}$ can, by a suitable choice of the constants of projection, be written :

$$
x^{2} z+y^{2} x+z^{2} y+2 \eta x y z=0,
$$

the other sheaf of cubics in-circumscribed in this same triangle being

$\left[\mathbf{A}^{\prime}\right]$

$$
y^{2} z+z^{2} x+x^{2} y+2 \eta x y z=0,
$$

* This equation will be referred to indifferently as sheaf [A] or cubic (of sheaf) [A]. 
where $\left[\mathrm{A}^{\prime}\right]$ is derived from $[\mathrm{A}]$ by the involutory transformation

$$
x: y: z=y^{\prime} z^{\prime}: x^{\prime} z^{\prime}: x^{\prime} y^{\prime} .
$$

The two cubics $[\mathrm{A}]$ and $\left[\mathrm{A}^{\prime}\right]$ are evidently transformed into themselves by the three transformations :

$$
x: y: z=y^{\prime}: z^{\prime}: x^{\prime}, \quad z^{\prime}: x^{\prime}: y^{\prime}, \quad x^{\prime}: y^{\prime}: z^{\prime} ;
$$

the remainder of the 18 linear transformations of $C_{3}$ into itself depend on the parameter $\eta$. If a straight line be drawn through any vertex of the triangle of reference and the two variable points of intersection with $C_{3}$ be put in correspondence we have the three involutory transformations of $[A]$ or $\left[A^{\prime}\right]$ into themselves given by

$$
x: y: z=y^{\prime} z^{\prime}: x^{\prime} y^{\prime}: x^{\prime} z^{\prime}, \quad x^{\prime} y^{\prime}: x^{\prime} z^{\prime}: y^{\prime} z^{\prime}, \quad x^{\prime} z^{\prime}: y^{\prime} z^{\prime}: x^{\prime} y^{\prime} .
$$

Under transformation [C] the nine inflexion points will be interchanged with the vertices of three in-circumscribed triangles and all the remaining vertices of incircumscribed triangles will be merely interchanged. Thus, of the covariants whose vanishing gives the vertices of the triangles in question, three will go over into the sides of the associated inflexion triangle and must therefore be conics circumscribing the triangle of reference, while the remaining covariants will be merely interchanged or transformed into themselves under the transformation [C].

To obtain these conics it will thus only be necessary to determine the equation of the sides of the associated inflexion triangle.

The Hessian of $[\mathrm{A}]$ is

$$
x^{3}+y^{3}+z^{3}-3 x y z-\eta^{2}\left(x^{2} z+y^{2} x+z^{2} y+2 \eta x y z\right)=0 ;
$$

thus all the cubics of either sheaf $[\mathrm{A}]$ or $\left[\mathrm{A}^{\prime}\right]$ have the fixed inflexion triangle:

$$
x^{3}+y^{3}+z^{3}-3 x y z=\left|\begin{array}{lll}
y & z & x \\
x & y & z \\
z & x & y
\end{array}\right|=(x+y+z)\left(x+\epsilon y+\epsilon^{2} z\right)\left(x+\epsilon^{2} y+\epsilon z\right),
$$

where $\epsilon^{3}=1$. By the application to this inflexion triangle of the transformation $[\mathrm{C}]$ we find that the vertices of three of the in-circumscribed triangles of the sheaves $[\mathrm{A}],\left[\mathrm{A}^{\prime}\right]$ lie on the conies:

$$
\begin{aligned}
& x y+y z+x z=0, \\
& x y+\epsilon y z+\epsilon^{2} x z=0, \\
& x y+\epsilon^{2} y z+\epsilon x z=0 .
\end{aligned}
$$

Each of these conics has a double contact with two sides of the associated inflexion triangle, the third side being the chord of contact. The Hessian of the net determined by $(a),(\beta),(\gamma)$ being the sides of the triangle of reference.*

* The conics $a, \beta, \gamma$ may be obtained directly without recourse to the Hessian ; for if $a x+b y+c z=0$ be the equation of a side of the associated inflexion triangle we must have, applying [C], $a x z+b y z+c x y \equiv \rho(a y z+b x y+c x z)$, so that $\rho=v^{3} 1$, i. e, $a=b=c$ or $a=\varepsilon b=\varepsilon^{2} c$ or $a=\varepsilon^{2} b=\varepsilon c$. 
The vertex of any in-circumscribed triangle is the tangential of three points whose affixes are given by $\bar{u}=\left(n \omega+n^{\prime} \omega^{\prime}\right) / 18$. The loci of the nine points associated with the vertices of the triangle of reference on which the $\bar{u}$ points lie three by three are :

$$
\begin{aligned}
& x^{2}-y z=0, \\
& y^{2}-x z=0, \\
& z^{2}-x y=0 .
\end{aligned}
$$

These conics are the first polars of the vertices of the fundamental triangle relative to the inflexion triangle, and have precisely the same relation to the fundamental triangle that $(a),(\beta),(\gamma)$ have to the inflexion triangle. The Hessian of their net is thus the sides of the inflexion triangle. If the inflexion triangle be taken as the triangle of coördinates, $[\mathrm{A}]$ can be written :

where

$$
\bar{x}^{3}+\bar{y}^{3}+\bar{z}^{3}+6 b \bar{x} \bar{y} \bar{z}=0,
$$

$$
\begin{aligned}
& \bar{x}=\sqrt[3]{3+2 \eta}(x+y+z), \\
& \bar{y}=\sqrt[3]{3 \epsilon^{2}+2 \eta}\left(x+\epsilon^{2} y+\epsilon z\right), \\
& \bar{z}=\sqrt[3]{3 \epsilon+2 \eta\left(x+\epsilon y+\epsilon^{2} z\right),} \\
& b=-\frac{\eta}{\sqrt[3]{2} 27+8 \eta^{3}} .
\end{aligned}
$$

Writing

$$
\lambda_{1}=\sqrt[3]{3+2 \eta}, \quad \lambda_{2}=\sqrt[3]{3 \epsilon^{2}+2 \eta}, \quad \lambda_{3}=\sqrt[3]{3 \epsilon+2 \eta},
$$

we have, as the equation of a side of one of the variable inflexion triangles of [A] ,

$$
\left(\lambda_{1}+\lambda_{2}+\lambda_{3}\right) x+\left(\lambda_{1}+\epsilon^{2} \lambda_{2}+\epsilon \lambda_{3}\right) y+\left(\lambda_{1}+\epsilon \lambda_{2}+\epsilon^{2} \lambda_{3}\right) z=0,
$$

which envelopes the locus :

or

$$
\begin{aligned}
& \rho u=\lambda_{1}+\lambda_{2}+\lambda_{3}, \\
& \rho v=\lambda_{1}+\epsilon^{2} \lambda_{2}+\epsilon \lambda_{3}, \\
& \rho w=\lambda_{1}+\epsilon \lambda_{2}+\epsilon^{2} \lambda_{3},
\end{aligned}
$$

$$
w v^{2}+v u^{2}+u w^{2}=0 \text {. }
$$

This equianharmonic curve of class three is inscribed in all three of the variable inflexion triangles of $[\mathrm{A}]$.

The locus of the variable vertices of these inflexion triangles is found to be three sextics.

The equations of the nine joins of the vertices of the triangle of reference and those of the associated inflexion triangle are :

$$
\begin{array}{lll}
x-y=0, & x-\epsilon y=0, & x-\epsilon^{2} y=0, \\
y-z=0, & y-\epsilon z=0, & y-\epsilon^{2} z=0, \\
z-x=0, & z-\epsilon x=0, & z-\epsilon^{2} x=0 .
\end{array}
$$


Since any three of these equations, picked out after the manner of a term of a three-row determinant, are simultaneous we have the theorem :

Each in-circumscribed triangle is in six ways perspective with its associated inflexion triangle. *

There remain two more triangles associated with the inflexion triangle under consideration. The loci of these vertices are easily determined by the method employed by Halphen. Let $x^{\prime} y^{\prime} z^{\prime}$ be the current coördinates; the tangent at $x y z$ is

$$
x^{\prime}\left(2 x z+y^{2}+2 \eta y z\right)+y^{\prime}\left(2 x y+z^{2}+2 \eta x z\right)+z^{\prime}\left(2 y z+x^{2}+2 \eta x y\right)=0 .
$$

If we make the substitution $x^{\prime}: y^{\prime}: z^{\prime}=z: x: y$ which transforms [A] into itself, it is clear that the locus :

$$
z\left(2 x z+y^{2}+2 \eta y z\right)+x\left(2 x y+z^{2}+2 \eta x z\right)+y\left(2 y z+x^{2}+2 \eta x y\right)=0,
$$

will pass through the vertices of an in-circumscribed triangle. Eliminating $\eta$ by means of $[\mathrm{A}]$ we get :

that is,

$$
y^{4} z^{2}+z^{4} x^{2}+x^{4} y^{2}-z^{3} x y^{2}-x^{2} y^{3} z-x^{3} y z^{2}=0,
$$

$$
\left(y^{2} z+\epsilon z^{2} x+\epsilon^{2} x^{2} y\right)\left(y^{2} z+\epsilon^{2} z^{2} x+\epsilon x^{2} y\right)=0 . \dagger
$$

These two equianharmonic cubics are in-circumscribed in three of the inflexion triangles of the sheaf :

$$
x^{3}+y^{3}+z^{3}+6 b x y z=0,
$$

and have the fourth inflexion triangle for an inflexion triangle.

It is to be noted that the conics $a, \beta, \gamma, a^{\prime}, \beta^{\prime}, \gamma^{\prime}$ are taken together a combinant of the sheaf of cubics [D], there being in all thirty-six such conics, each of which circumscribes one inflexion triangle and has a double contact with two sides of another, the third side of the second triangle being the chord of contact. The rôle played by these conics in the geometry of the sheaf [D] has not, so far as the writer is aware, been investigated.

Yale University, New Haven, Conn.

* The same thing is well known to be true for any two inflexion triangles, the six centers of perspectivity here being the vertices of the other two inflexion triangles. The theorem in question may be deduced from the fact that the vertices of the inflexion triangles lie three by three on the harmonic polars.

† These cubics are together with $y^{2} z+z^{2} x+x^{2} y=0$ a combinant of the sheaf of curves [D] below and determine on it the vertices of nine in-circumscribed triangles. These cubics belong to the set of eight obtained by HALPHEN, loc. cit., and were given by Professor H. S. White, Transactions of the American Mathematical Society, April, 1900. 